\title{
A spatial data model for urban spatial-temporal accessibility analysis
}

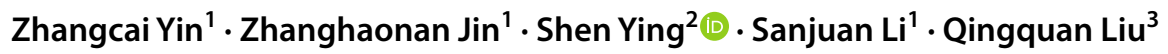

Received: 19 May 2019 / Accepted: 12 June 2020 / Published online: 29 June 2020

(c) Springer-Verlag GmbH Germany, part of Springer Nature 2020

\begin{abstract}
Time geography represents the uncertainty of the space-time position of moving objects through two basic structures, the space-time path and space-time prism, which are subject to the speed allowed in the travel environment. Thus, any attempt at a quantitative time-geographic analysis must consider the actual velocity with respect to space. In a trip, individuals tend to pass through structurally varying spaces, such as linear traffic networks and planar walking surfaces, which are not suitable for use in a single GIS spatial data model (i.e., network, raster) that is only applicable to a single spatial structure (i.e., point, line, polygon). In this study, a velocity model is developed for a traffic network and walking surface-constrained travel environment through the divide-and-conquer principle. The construction of this model can be divided into three basic steps: the spatial layering of the dualconstrained travel environment; independent modelling of each layer using different spatial data models; and generation of layer-based time-geographic framework by merging models of each layer. We demonstrate the usefulness of the model for studying the space-time accessibility of a moving object over a study area with varying spatial structures. Finally, an example is given to analyse the effectiveness of the proposed model.
\end{abstract}

Keywords Time geography $\cdot$ Spatial data model $\cdot$ Layer $\cdot$ Velocity $\cdot$ Accessibility

JEL Classification 2.200

\section{Introduction}

Over the past decade, there has been rekindled interest in the Hägerstraand (1970) time-geography methodology through its application to the area of quantitative geographic analysis (Miller 2005). Quantitative time-geographic analysis seeks

Shen Ying

shy@whu.edu.cn

Extended author information available on the last page of the article 
to identify all the locations in space-time that a mobile object can occupy during an episode of potential movement, given the maximum velocities in the travel environment (Kuijpers et al. 2010; Kobayashi et al. 2011). The maximum velocity (denoted $v_{\max }$ ) affects the accessibility of space-time locations to individuals, which, in turn, affects the time-geographical structures that represent those possible locations. For instance, $v_{\max }$ is inversely proportional to the slope of its space-time path (STP), a basic tenet of time geography (Delafontaine et al. 2011), whereas it is directly proportional to the cone angle of the space-time prism, a central concept in time geography and (increasingly) of mobile object databases (Kobayashi et al. 2011). This indicates that time geography with respect to measures for space-time accessibility is highly sensitive to $v_{\max }$; hence, any type of quantitative time-geographic analysis must consider the actual $v_{\max }$ allowed in the environment.

Classical time geography assumes that $v_{\max }$ is only related to the movement capabilities of an individual; therefore, it can be treated as constant or fixed (Downs and Horner 2014). This assumption presents a strong bias for the time-geographic analysis (Liao 2019). More specifically, it exaggerates the prism if $v_{\max }$ is set too large and fails to capture visited areas if $v_{\max }$ is set too small (Loraamm et al. 2018). For this reason, the heterogeneity of $v_{\max }$ has been the focus in time geography studies, concentrating on features such as time-dependent transport networks (Arentze and Timmermans 2004; Neutens et al. 2011; Fayyaz et al. 2017), supernetworks (Liao 2019), and adaptive velocity in reachability analysis (Downs and Horner 2014). Heterogeneity is not only reflected in the time dimension and dimensions of behaviour patterns but also, most commonly, in the spatial dimension; that is, $v_{\max }$ is not always equal in space. Relative to the linear time dimension, space is flat or threedimensional, and it carries moving objects for any moment or behaviour mode. This means that recording a non-uniform $v_{\max }$ distributed over space is the basis for the evolution of time geography from classic to modern.

Recent advances in time geography offer new perspectives for modelling structural heterogeneity that impede movement potential. These advances have been shown to produce more realistic results for time-geographic methods (Loraamm et al. 2018). Prior to these developments, Miller and Bridwell (2009) pioneered the introduction of field-based time geography, which considers limitations in movement potential (i.e., traffic network and walking surface) as a spatial field (Long 2018). A GIS field is a conceptual model that is often used to describe the variable properties at each location. Here, the velocity field essentially converts various spatial limitations into a unified measurement index, that is, the upper bound of the maximum possible travel velocity allowed by each spatial location to provide a computable spatial data model for the cost-distance analysis of GIS. In addition, the velocity field degenerates into a uniform velocity distribution assumed by classical time geography under a homogeneous environment. This approach is advantageous in that it directly considers movement cost surfaces in the calculation of a potential STP (Long and Nelson 2013) and allows the examination of theoretical conjectures regarding accessibility in continuous space. Time geography, in line with the trend in quantitative development, necessitates the representation of a location-varying $v_{\max }$ through a spatial data field model. 
Thus far, however, the velocity field in time geography has only been implemented as a raster grid (Goodchild 1977; Upchurch et al. 2004; Miller and Bridwell 2009; Delafontaine et al. 2011; Yin et al. 2013; Long and Nelson 2013; Long 2016; Loraamm 2019). This raster-based velocity field is limited in that the spatial decomposition of the transportation network into a lattice is required to use this approach in empirical research (Delafontaine et al. 2011). Replacing network paths with lattice paths results in position deviation error (Goodchild 1977) and spatial relationship error. From the perspective of GIScience, spatial relationship error can be described by the following aspects. (1) The lattice path can approximate the route with reasonable spatial granularity; however, it does not converge to a continuousspace route, resulting in the overestimation of the network path (also known as elongation error, Goodchild 1977) or underestimation in the distance (Delamater et al. 2012). (2) Another drawback is that the raster model is ill-suited for dealing with complex network topologies, such as bridges and tunnels, one-way streets, and turn prohibitions (Neutens et al. 2011; Delamater et al. 2012). For example, two colocated roadways, such as two non-interconnected viaducts and tunnels, are always regarded as connected to each other at co-located cells, or two roadways on two adjacent cells are assumed to be interconnected at adjacent cells. (3) Concerning the spatial direction, the isotropic raster cell generalises the velocity vector along the road. Consequently, a raster-based velocity field is likely to contain areas never visited by the object (commission error) and miss actual visited locations (omission error) (Sanderson 1966; Long and Nelson 2013). These errors propagate to the prism and then to the prism-prism intersections. Similar results were also observed in other studies (Kuijpers et al. 2010; Kobayashi et al. 2011; Leung et al. 2016; Long 2018). Arguably, however, the errors caused by these methodological issues cannot be resolved by increasing the lattice density (Delafontaine et al. 2011).

To alleviate these limitations, this article proposes layer-based time geography as a new tool to build the velocity field for network and nonnetwork-constrained travel environments (e.g., urban locales). Mathematically, the velocity field is a smooth differential function that assigns a velocity to each location in continuous space (Delafontaine et al. 2011). The approach presented in this research follows GIScience by factoring in the spatial characteristics of each data layer in travel space, such that each layer can match the appropriate spatial data model from GIS. This being the case, the respective models corresponding to each layer are aggregated into a layered velocity field, which is different from similar approaches using a single spatial data model, e.g., a raster grid to represent layers with varying spatial types indiscriminately. The aim of the present study is to enhance the applicability of velocity field theory to network- and nonnetwork-constrained environments and put it into practice by proposing and implementing a formal theoretical framework for defining and constructing hierarchical time geography. The framework is particularly useful for modelling movement that occurs in a constrained space (e.g., citizens travelling in urban locales) and accounts for both path and accessibility errors.

The remainder of this article is organised as follows. Since our approach relies on GIScience, the next section introduces the key data models of GIS and documents the velocity models that have been developed in recent years for cost-distance analysis in time geography. Then, in Sect. 3, both velocity models are combined, and the 
outcome is a model for representing the velocity field with network and non-network constraints. The model is implemented and tested in urban locales in Sects. 4 and 5. Conclusions close the article in Sect. 6.

\section{Background}

As the constriction of movement possibilities leads to more realistic analytical results rather than purely a conceptual value, significant attention has been given toward removing the unrealistic assumption of isotropic speed in an unconstrained space in the traditional time-geographic framework (Versichele et al. 2014). The removal of this assumption was performed by means of GIS data models to deal with anisotropy speed in a constrained environment (Miller 1991; Shaw and Yu 2009). To that effect, there have been two fundamentally different approaches presented in the literature: the network-based method (Kwan and Hong 1998; Kuijpers and Othman 2009; Kuijpers et al. 2010) and the raster-based method (O'Sullivan et al. 2000; Upchurch et al. 2004; Miller and Bridwell 2009; Long 2016). These two models typically belong to the two basic models of network and raster in GIS. Because each of the network and raster models has its own advantages, there is a growing demand for the further integration of these two models. Layer-based time geography, developed below with the discussion presented in the next section, considers the combination of these two models. As follows, we focus on the aspects of time-geographic research that are most relevant to the development of such a layerbased approach.

\subsection{Network-based velocity model}

Time geography considers link-based travel speeds in a transportation system used to model any moving object present in context with roadway networks (Miller 1991). The transportation networks can be represented through a network data model in GIS, in which each arc is assigned a maximum attainable velocity that varies by arc (Kuijpers and Othman 2009). Further, the labelling speed limit $v_{\max }$ of each arc in the network as described can be converted to an associated time span in the following way - the length of the arc is divided by $v_{\max }$, in which the time span is the least time-cost required to go from one side of an arc to another when travelling at the speed limit (Kuijpers et al. 2010).

From the traffic network with defined travel costs, accessibility analysis can be developed using well-established methods. The least-cost path can be computed between any two points on the network using a path optimisation algorithm (e.g., Dijkstra's algorithm). Given a road network, starting point $s$, any point $p$ on the network, and travel time budget $\tau$, we can determine whether $p$ is reachable in this way: if the least travel time between $s$ and $p$ (denoted $t_{s p}$ ) is no greater than $\tau$, then $p$ is reachable. All reachable points $\{p\}$ make up the reachable subnet. When an end point $e$ is also known, such reachable points $\{p\}$ satisfy: $t_{s p}+t_{p e} \leq \tau$. 
Furthermore, these reachable points $\{p\}$, as a part of the road network, can generate isochrones (lines of equal travel time) through spatial interpolation (O'Sullivan et al. 2000), which includes not only the networks but also the non-network environments (e.g., walking surface). However, the neglect of the contextual off-traffic network produces errors in the isochrone method, such as commission and omission errors, which are usually very serious in areas with sparse traffic networks (Upchurch et al. 2004). Therefore, time geography on and off roadways needs to consider the impact of joint constraints of the road network and pedestrian surfaces on individual movement; this also implies that both car and walking modes need to be considered.

As an extension of the conventional network model, the supernetwork can be used to construct multi-modal, multi-activity, time-dependent tours (trip chains) (Arentze and Timmermans 2004; Liao 2019). To that end, the supernetwork model uses different copies of the same original physical transportation network to express different activity states or transit time periods (e.g., peak and off-peak periods) (Arentze and Timmermans 2004), or integrates different networks of different traffic modes (Carlier et al. 2003). Whereas, these supernetworks, like the general network model, focus on network constraints, which is consistent with most traffic modes that are only restricted by the network. However, for some modes of transportation (e.g. walking), the constraint space involves an area component (e.g., walkable squares) as well as a network component (e.g., walkable paths). For such a traffic mode, both components need to be considered simultaneously in the spatial data modelling for space-time accessibility analysis. Area-dependent spatial data are usually expressed in GIS, using the conceptual field model.

\subsection{Raster-based velocity model}

To remedy the shortcomings of multi-mode networks or supernetworks in expressing traffic modes involving areas, field-based velocity surface models have emerged. However, their raster implementations bring about position deviation error and spatial relationship error when modelling the traffic network. These errors are unevenly distributed in space; for example, the errors in areas with dense traffic networks (e.g., metropolitan) are usually smaller than those in areas with sparse traffic networks (e.g., sub-metropolitan areas, areas away from the central business district) (Apparicio et al. 2008). Further, using these raster data to analyse accessibility can introduce commission and omission errors into the results.

Following GIScience, the Miller and Bridwell (2009) velocity field realise a unified expression for the walking area and the network for cars; however, its raster-based implementation, like other field models, cannot escape the errors mentioned above. The most relevant details for this field are repeated here for convenience. We clarify the approach using a simple example case. Figure 1a shows a $30 \times 30 \mathrm{~km}$ map with two streets, marked as $R_{1}$ and $R_{2}$. The area surrounding the streets can be assumed to be open and accessible to pedestrians. Let the speed in the streets and pedestrian zone be $10 \mathrm{~km} / \mathrm{h}$ and $5 \mathrm{~km} / \mathrm{h}$, respectively. We can build a raster model with nine cells, each with a spatial extent of 


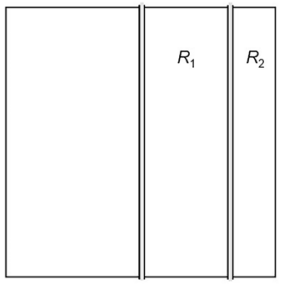

(a)

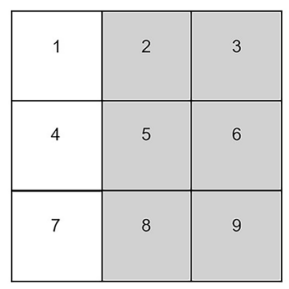

(b)

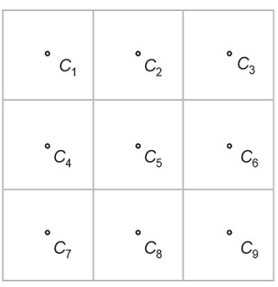

(c)

Fig. 1 a Field with roads R1 and R2, b its raster model, and c centre-point model

$10 \times 10 \mathrm{~km}$ and a velocity $v_{\max }$ to cross (Fig. 1b). Thus, the speeds of the pixels that are spatially coincident with roads are defined as $10 \mathrm{~km} / \mathrm{h}$ and $5 \mathrm{~km} / \mathrm{h}$, elsewhere. As argued by Downs et al. (2014), all calculations are performed at the cell centroids, with the resulting values generalised to the rest of the cell. Toward this end, the raster model (Fig. 1b) can be converted into nine centroids $\left(C_{1}, C_{2}, \ldots, C_{9}\right.$, Fig. 1c).

In line with the general rasterization of network data, the raster model of Fig. 1 will bring the aforementioned errors. Taking the least travel cost (in units of time) to go from $C_{5}$ to $C_{6}$ as an example, pursuant to this model in Fig. $1 \mathrm{~b}$, the cost can be calculated as the following: the distance between $C_{5}$ and $C_{6} \div$ the speed of $10 \mathrm{~km} / \mathrm{h}=10 \mathrm{~km} \div 10 \mathrm{~km} / \mathrm{h}=1 \mathrm{~h}$. Rather than $1 \mathrm{~h}$, this actual cost is greater than $1 \mathrm{~h}$, and up to $2 \mathrm{~h}$ (i.e., $10 \mathrm{~km} \div 5 \mathrm{~km} / \mathrm{h}$ ), as there is no direct road traversing between $C_{5}$ and $C_{6}$ (Fig. 1a). This error arises from the generalisation of the velocity along the road to all directions in the grid cell; consequently, the horizontal direction (that is, the line segment with endpoints $C_{5}$ and $C_{6}$ ) also has car speed instead of actual walking speed. As the example illustrates, this rasterization of network data comes at the cost of a considerable increase in errors, such as the over- or underestimation of travel time (Delamater et al. 2012). In general, in terms of measuring accessibility, network distances provide more appropriate results than other distance types, such as Manhattan distance (Apparicio et al. 2008) and lattice distance. This implies that both network data and surface data need to be expressed separately using their most suitable data structures, namely the network model and raster model.

Additionally, as the Miller and Bridwell (2009) empirical studies indicated, some traffic modes (e.g., walking, biking) include not only network-like but also plane-like characteristics. To date, existing supernetwork models consider the physical transport network exclusively, limiting their relevance for traffic models involving a planar structure (e.g., walking area). To circumvent this problem, prior velocity-field models represent the area part of the walking mode well but sacrifice the physical network of the car mode. The aim of this research is to fill this gap by absorbing their advantages: the networked representation of existing velocity-field models to planar regions and the full inclusion of the supernetwork to the original physical network. 


\section{Layer-based velocity model}

In this section, a divide-and-conquer technique is proposed as a possible alternative to the raster-based modelling method in the context of a network and nonnetwork travel environment. Divide-and-conquer algorithms attack a complex problem by dividing it into simpler problems whose solutions can be combined to yield a solution to the complex problem; this approach can often lead to simple, elegant, and efficient algorithms (Jordan and Jacobs 1994). We describe layerbased velocity field architecture, which is inspired by the divide-and-conquer philosophy. The algorithm for the framework can conceptually be divided into three steps: (1) decompose a travel environment into two layers-the traffic network and walking surface; (2) establish an independent speed model for each layer; and (3) integrate these obtained models to constitute a complete velocity model for the travel environment. In theory, this method can simplify the complexity of velocity field modelling and complement the advantages of different spatial data models in GIS. The output of the method consists of two interconnected networks representing the traffic network and the walking surface separately. A comprehensible methodology for the construction of a layer-based velocity model is elaborated at length.

\subsection{Layering of travel environment}

As argued before, in real life, the travel context is often not just network- or nonnetwork-constrained but both. For example, in a tour, students walked away across the campus where taxis were restricted and then took taxis on the off-campus roads. To cope with both network and non-network constraints in most realworld applications, we introduce a divide-and-conquer strategy.

A constrained environment, for convenience of explanation, can be exemplified as such a velocity field that includes a roadway network and a walking surface. The first step in building a spatial data model associated with multiple modes is to construct each spatial data model associated with a single mode, which leads to a divide-and-conquer strategy. In the GIS field, this strategy has been applied to the hierarchical organisation of spatial data in certain ways, e.g., spatial geometry types. Here, "divide" is to section the travel space into two layers, the traffic network and walking surface, in terms of the line and polygon feature classes, respectively (Fig. 2a). A layer, from the GIScience perspective, is a collection of spatial objects with the same feature classes. Apart from geometric types, attribute types of geographical features can serve as stratification of the spatial data sets as well.

The idea of layering was also reflected in supernetworks (e.g., Carlier et al. 2003; Arentze and Timmermans 2004; Liao 2019), where a copy of the traffic network corresponding to a traffic mode can be considered a layer. This proposed method, like the Miller and Bridwell (2009) model, considers the area-related traffic mode (walking) and is, therefore, different from supernetworks, which focus on network constraints. 


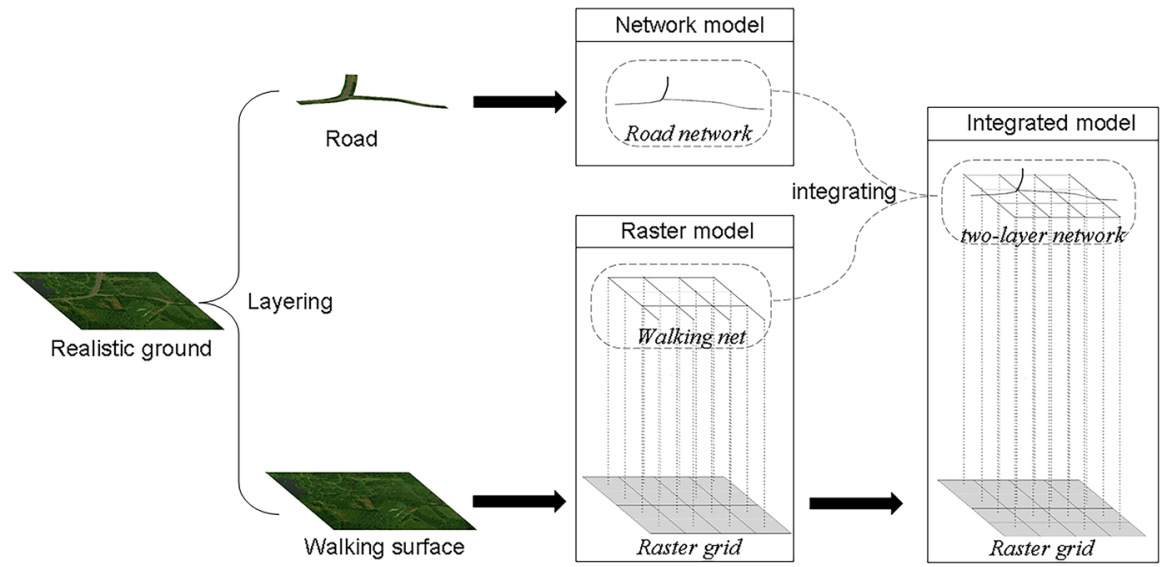

Fig. 2 Layer-based time geography: a "divide", b "conquer", and c "integrate"

\subsection{Velocity modelling of travel environment layers}

Development in time geography draws heavily on well-established methods for modelling spatial data, common to most GIS software packages. In the research, "conquer" refers to a data layer corresponding to a spatial data model to ensure the independence and rationality of the expression of each layer (Fig. 2b). In such a case, a data model employed by one layer can be separated methodologically from that employed by the other. Specifically, two different logical structures, namely, the GIS network model and raster model, are adopted to represent the road network and walking surface in the travel environment, respectively. Although the constraints on individual movement are diverse in terms of types, they can be regarded as non-spatial attributes from the perspective of GIS data type and can be uniformly converted into the velocity attributes over space.

\subsubsection{Modelling the velocity layer for car mode}

As with the general network-based method, the transportation network layer can be represented as a velocity network with either uniform speed limits over the entire network or speed limits that may vary per arc and for bi- or uni-directional arcs (Kuijpers et al. 2010), which provides the maximum possible velocities associated with each location in transportation-network space. The classical traffic-network model can be adapted to handle a multi-modal supernetwork with minor changes (Carlier et al. 2003).

\subsubsection{Modelling the velocity layer for walking mode}

Despite the proliferation of methods to delineate travel possibilities within traffic networks, only a few studies have been concerned with modelling non-motorised, 
non-network yet spatially constrained movements (Delafontaine et al. 2011). Walking belongs to this category; that is, it is not confined to network spaces, such as that taking place in parks, college campuses, or other open areas where pedestrians are not restricted to sidewalks or roadways (Downs and Horner 2014). Existing supernetworks represented the walking mode but only the physical network part of the mode, while ignoring the area part (e.g., Arentze and Timmermans 2004; Lee and Miller 2018). An exception may be Miller and Bridwell (2009), which suggest an analytical theory to model velocity fields in the context of traffic networks and walking surfaces. Although, products yielded from this research is not specifically designed for dealing well with non-network walking surfaces, this theory, as the marriage of time geographic theory and popular GIS methods, can be used as a velocity field for a walking surface in such a specific situation, in which the speed of the traffic network is zero or there is no network in the study area. Moreover, this theory has been recently applied to some fields, such as in ecology to estimate home ranges (or habitats) and utilisation distributions (or movement probabilities) and in transportation to generate isochrones (Long 2016, 2018). In this sense, the time geography of Miller and Bridwell (2009) is applicable to non-network spaces (e.g., walking surface) embedded in human movement, including an orienteering event and the potential movements of the missing person and recreationalists (e.g., hunters and hikers) (Long 2018).

Walking is a ubiquitous mode of transportation. It is the first choice for shortdistance transportation (especially no more than $1 \mathrm{~km}$, Shelat et al. 2018) or nonmotorised areas (e.g., city squares). In addition, walking is an important bridge connecting different traffic modes in multi-mode traffic, serving as "glue" (Carlier et al. 2003). Although walking has a small or negligible share of the distance- or time-cost in long-distance journeys between cities, this is not necessarily the case in urban areas. Even in cities with dense transportation networks, motorised traffic is not always better than foot traffic in terms of time-cost, owing to the many restrictions on traffic, such as the prohibition of left turns, one-way traffic, congestion, residential fences, and limits in numbers. Furthermore, walking can even become an important way for individuals to travel under some special circumstances, such as during the outbreak of infectious diseases (e.g., COVID-19) when public transport is temporarily closed and private cars are restricted. These discussions show that walking is an indispensable way of multimodal transportation. Consequently, focusing on space-time accessibility and its boundaries, time geography needs to consider the impact of walking on accessibility, whether in areas with sparse traffic networks and in cities with dense traffic networks.

The walking surface can be represented as a velocity field, which provides maximum velocities associated with every location in walking space. It can be isotropic where local velocities are unidirectional (e.g., walking through thick forest) or anisotropic where local velocities are direction specific (e.g., walking up or down a hill), and also can capture both hard (e.g., lakes) and soft (e.g., variations in topography and land cover) barriers to movement (Long 2018). As noted by Dykes and Mountain (2003), the raster-based implementations of the walking-velocity field can be performed by subdividing a study area into a lattice (commonly a raster) and determining $v_{\max }$ of each cell. The outcome is a heterogeneously weighted spatial lattice. 
Because physical barriers and obstacles associated with lakes, fences, and speedrestricted zones can all be incorporated into the generation of a suitable velocity surface, the velocity of the cell ranges from zero (obstruction-filled unit) to an upper bound on the theoretical maximum walking velocity (obstruction-free unit), with intermediate values reflecting the velocity that is allowed in a unit partially filled with obstacles.

The measures of cost distance are commonly quantified using a node-link representation (Long 2018). With raster datasets, a node represents a cell in a two-dimensional regular lattice, and a link makes a connection between a cell and its neighbour (Long 2018), along with a move permitted in the lattice. Typically in this type of analysis, moves from each cell are limited to the four or eight cardinal directions (i.e., rook- or queen-case definition of neighbours, Fig. 3); however, other definitions of neighbourhood types are possible (Goodchild 1977; Collischonn and Pilar 2000; Miller and Bridwell 2009; Gonçalves 2010; Long 2018). As such, the raster-based walking velocity field can be converted into the network-based structure, hereafter referred to herein as the neighbour network, transforming the reachability analysis on a regular lattice into STP analysis on the network. Prior to network analysis, the neighbour network needs to be given weights, such as speed.

The neighbour network is structurally derived from the raster grid, and its weight is the same. Each cell $c$ in the raster grid is indexed as $i$ with velocity $v_{i}$ and centroid $n_{i}$ serving as a node in the neighbour network. Cells next to $c_{i}$ are denoted $c_{j}$ with velocity $v_{j}$ and centroid/node $n_{j}$. Let $v_{i j}$ denote the velocity weight of the link $n_{i} n_{j}$ connecting nodes $n_{i}$ and $n_{j}$, whose value is equal to the length of $n_{i} n_{j}$ divided by the minimum time cost to pass it. For different neighbourhood types (e.g., fourcell, eight-cell), the formula for $v_{i j}$ is different. Given a square cell with a length of $2 a$, taking the four-cell pattern as an example, the calculation process for $v_{i j}$ can be divided into two parts: calculate the length of $n_{i} n_{j}$ as $2 a$ and calculate the minimum elapsed time through $n_{i} n_{j}$ as $\left(a / v_{i}+a / v_{j}\right)=a\left(v_{i}+v_{j}\right) / v_{i} v_{j}$. In this vein, $v_{i j}$ can be mathematically formulated as:

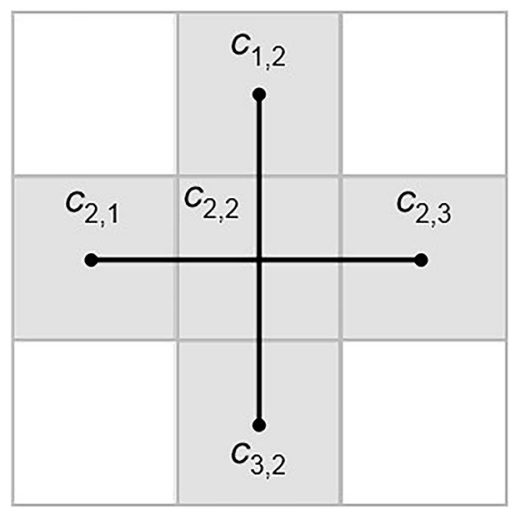

(a)

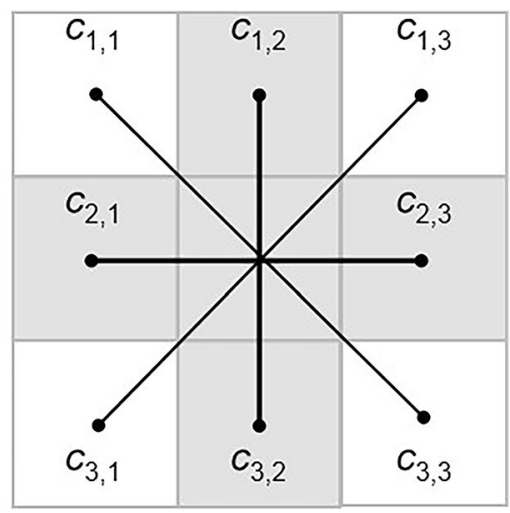

(b)

Fig. 3 Two neighbourhood types in raster grid: a four-cell (rooks); b eight-cell (queens) 


$$
v_{i j}=\frac{2 \cdot v_{i} \cdot v_{j}}{v_{i}+v_{j}},
$$

where $v_{i j}$ is the speed limit for link $n_{i} n_{j}$. While different in their respective implementations, the neighbour network and its alternative raster model represent different approaches to the same question, dealing with the representation of velocities over a walking surface and a map to each other in spatial position and velocity properties.

In addition, akin to the conclusion that the Manhattan distance is close to the network distance in areas with dense networks (Apparicio et al. 2008), the actual walkway in areas with dense walkways can be approximated by the lattice path, in theory. This means that for areas with dense walkways, the raster grid is a very reliable alternative to actual trail networks. In addition, the neighbour network and the actual trails located inside and outside the walking zone together constitute the walking net for the walking mode.

\subsection{Combining velocity models of travel environment layers}

In this section, we further introduce the integration of the models of Sects. 3.2.1 and 3.2.2, which constitute the building blocks of a layer-based velocity model (Fig. 2c). The implementation of this integration is to add nodes as transfer links at some intersection between the car network and walking net; the outcome is a two-layer framework that combines the unimodal networks for walking and vehicle and includes transfer links for purposes of interconnection. Here, each new node coincides with a location in space, such as a taxi station and parking lot. This framework retains the car network and is, therefore, different from the Miller and Bridwell (2009) model, in which the car network is replaced by an artificial grid.

As Arentze and Timmermans (2004) stated when using a transfer link, the location of a moving object does not change; however, the mode of transport is shifted from one to another. Then, a path through the two-layer network, including such a transfer link, models a tour that contains two modes of trips and one or more mode switches. The two-layer network allows one to allocate time costs to the transfer links; however, the costs can be ignored in situations. For instance, time geography that seeks to maximize space-time accessibility lacks sufficient transferred time-cost data. The speed weights of the sub-arcs generated by the splitting of the new nodes can inherit the speed of the original arc. Consequently, the weighted two-layer network as described can be seen as a network version of the velocity field or surface, where the maximum velocities associated with each location in the environment can be recorded and, accordingly, can serve as the basis for cost-distance and accessibility analyses.

\section{Methods}

For illustration, the suggested approach is applied to urban settings for modelling the time-cost surface. The approach is executed using ArcGIS 10.2 with four cardinal neighbours, a raster resolution of $5 \mathrm{~m}$, and spatial extent of $2.7 \mathrm{~km} \times 2.7 \mathrm{~km}$. 
The study area includes Wuhan University of Technology (WHUT) and represents a constrained travel environment exhibiting the presence of roads and pedestrian zones, providing an interesting substrate upon which to demonstrate the approach. Road network data modified by OSM (https://www.openstreetmap. com) were imported into ArcGIS and represented as an undirected graph with time-invariant speeds. From the perspective of GIS analysis, roads are divided into three types: residential street, highway, and expressway, and their corresponding maximum speeds are set to 20,40 , and $60 \mathrm{~km} / \mathrm{h}$, respectively. The construction of a walking net can be described as follows:

1. Identifying a walking zone. The entire research area is cut by authority-constrained sub-areas, such as private residential areas, enterprises, and institutions, with the results of a walking zone. The zone, here the campus of WHUT, is then rasterised with 38,805 cells.

2. Assigning a walking speed to the walking zone. Each raster cell is given a speed attribute as per Tobler's Hiking function (Tobler 1993): speed $=6 \mathrm{e}^{-3.5|\tan \theta+.05|}$, where $\theta$ is the slope in degrees and speed is in $\mathrm{km} / \mathrm{h}$. Accordingly, walking paths outside the walking zone are assigned a speed of $5 \mathrm{~km} / \mathrm{h}$, as per Versichele et al. (2014).

3. Generating a walking net. This walking zone is first converted to a neighbour network with a four-cell pattern and then connected to walkways outside the walking zone, including footbridges and pedestrian tunnels, zebra crossings, and trails along the road, to create a pedestrian network.

Consequently, the road network and walking net can be interconnected in GIS software to generate a two-layer network with 121,587 nodes and 162,407 undirected links. For the campus, the neighbour network and residential streets are directly interconnected by new nodes at the intersections between them. For areas outside the campus, nodes are added at the interchangeable intersection-points between the trails and road network. Here, the trails are distributed along with the highway network. In the analysis of the least-cost path, because the speed of the highway is greater than that of the trail, the shortest path will pass through the highway network instead of the trails distributed along with the highway network. This hints that the trails accompanying the distribution of the road network can be omitted in the two-layer model for simplicity.

The two-layer network can serve as a basis for inferring potential space-time positions between observed anchors through cost-distance analysis using a secondary development of ArcGIS based on Python 2.7.1, provided that STP assumptions associated with the shortest path algorithm are satisfied by the behaviours of the individual in question. For this demonstration, a pair of known anchors located in WHUT, China, was designated by $s$ and $e$, which are the starting point (Nanhu New Campus Stadium) and end point (Jianhu No. 1 Teaching Building) with associated times of $t_{s}=0 \mathrm{~s}$ and $t_{e}=531 \mathrm{~s}$, respectively. The pair of anchors was chosen because they are located at different places for the two activities of physical education and self-study, and the traffic between the two points can be 
in vehicle mode, walking mode, or both. In addition, the time budget between $s$ and $e$ is usually set to 1.5 times the shortest time (354.2 s here) (Yin et al. 2020) or $531 \mathrm{~s}$. To do so, consider any intermediate time point $t$ between two anchors $\left\{s, t_{s}\right\}$ and $\left\{e, t_{e}\right\}$, where $t_{s}<t<t_{e}$. For a vertex $p$, two different time points are defined based on a two-layer network: $t_{p}^{-}$, which is the earliest possible arrival time from origin location $s$ to $p$, and $t_{p}^{+}$, which is the latest possible departure time from $p$ to destination location $e$ (Kuijpers et al. 2010). If $t_{p}^{-} \leq t \leq t_{p}^{+}, p$ is accessible at time $t$.

Using the ArcGIS Network Analyst further development based on Python, less than three days were needed to calculate $t^{-}$and $t^{+}$for each vertex on the two-layer network to complete on a normal laptop (Core ${ }^{\mathrm{TM}} \mathrm{i} 5-6200 \mathrm{u} 2.40 \mathrm{GHz}$ computer processor and $8 \mathrm{~GB}$ of RAM). Then, these accessible vertexes can be converted into the potentially accessible area at $t$, also termed an isochrone area (O'Sullivan et al. 2000) or disk (Downs et al. 2014), by mapping each vertex $p$ to a cell or road segment, depending on whether $p$ is a cell centroid or a road vertex. Moreover, the disks at each time $t$ together form a space-time prism with vertices $s$ and $e$ in the $(x, y, t)$-space, which maps the set of potential spatial locations for a mobile object over time, given known spatial, temporal, and physical constraints limiting their movements (Loraamm et al. 2018). When measuring reachability, the relationship between the spatial extent and the anchors needs to be considered. If the anchors are too far apart, the space-time disk will be truncated by the spatial extent with inaccuracy (Downs et al. 2014); otherwise, if they are too close, unnecessary data that must be stored outside the space-time disk will be increased, thereby contributing to the computational burden.

Finally, a direct comparison or validation confirming the utility or effectiveness for the layer-based methodology versus the pure raster-based one would be difficult to implement in a meaningful way, as these methodologies assume that the movement of an individual between any two point locations is only limited by the time cost. Meanwhile, the success of any lattice method is difficult to assess, as the continuous-space ideal is usually hypothetical (Goodchild 1977). Nevertheless, on a purely algorithmic level, we evaluated the performance of layer-based methodology by comparing its results (here, space-time disks) with the pure raster-based version. Parameters used for these two models include space-time anchors and speeds of different modes of transportation.

\section{Results}

Figure 4 illustrates the process of modelling the two-layer network for time-cost surface over the area concerning WHUT. According to the car and walking mode, this area is divided into two layers, which are depicted by Fig. 4a, b, respectively; Fig. 4c shows a part of the two-layer network. It is apparent from Fig. 4c that the two-layer network fully encompasses the physical road network and, consequently, can generate more realistic STPs. Moreover, each segment of such STPs can be practically surveyed and explained from the perspective of minimising resistance (i.e., time or effort) across a travel environment, which may open up opportunities for examining 


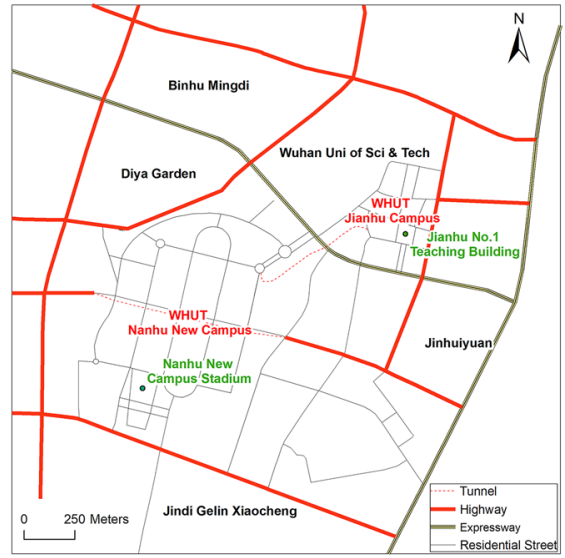

(a)

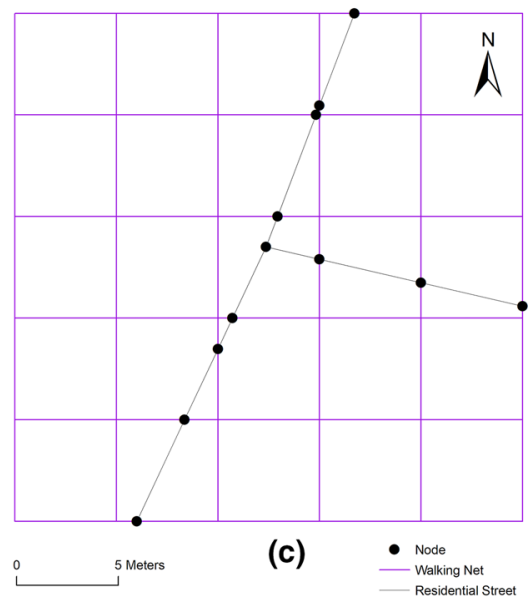

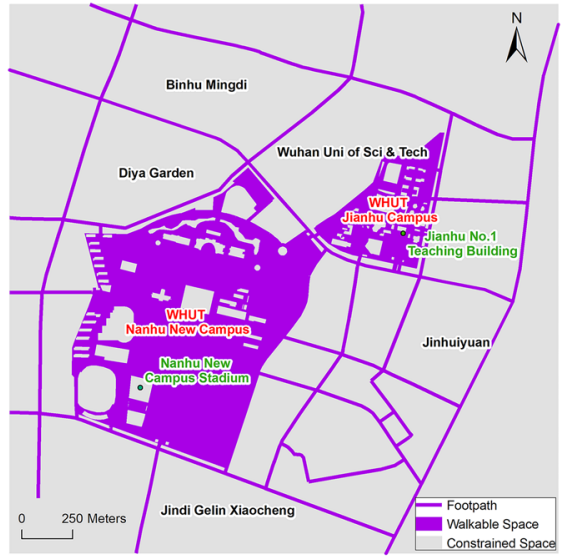

(b)

Fig. 4 Data models for $\mathbf{a}$ car mode, $\mathbf{b}$ walking mode, and $\mathbf{c}$ a mixture of the two

the results of the space-time accessibility analysis. The diagram also clearly showed that the walking net can evenly cover the walking zone, which can improve the accessibility evaluation in areas with sparse traffic networks. These analyses also mean that layer-based time geography is capable of identifying errors in other models of the velocity field. Notably, there are a large number of intersections between the roads and the walking net in Fig. 4c, which does not mean frequent transfers on each trip (this is not in line with common sense). Indeed, it can be known from the reachability measure method that a moving object from $s$ to $e$ always reaches point $p$ at $t_{p}^{-}$, stays at $p$ for as long as possible, i.e. $\left[t_{p}^{-}, t_{p}^{+}\right]$, and then leaves $p$ at time $t_{p}^{+}$. This means that the paths between $s-p$ and $p-e$ are based on least cost and, therefore, always follow the route with the highest speed possible, such as choosing a road instead of a pedestrian route. In short, the number of intersections characterises the degree of flexibility that maximises the time period of $\left[t_{p}^{-}, t_{p}^{+}\right]$for participating in the 
activity at $p$, making transfers less frequent for a trip unless such a situation as multiple activities arises.

Figure 5 demonstrates five space-time disks of a prism with anchors $\left\{s, t_{s}\right\}$ and $\left\{e, t_{e}\right\}$ in both space ( $X$ and $Y$ coordinates) and time ( $Z$ axis). Figure 5a visualises five blue-filled convex-shells associated with these disks and expresses the trend or overall overview of these disks over time. Furthermore, Fig. 5b shows that, visually or on map cognition, an unfilled convex-hull makes the parts in a space-time disk form a whole, which helps to distinguish the boundaries among the space-time disks. In addition, Fig. 5c shows the five disks of the prism at discrete time points: $81,181,281,381$, and 481 in s; each disk demarcates the spatial extent of the prism at corresponding points in time during its existence and can be seen as a spatial representation of limits of movement at the time associated with the disk (Long 2018). For simplicity, these space-time disks do not include accessible highway sections; existing research on the construction of space-time prisms has mainly focused on

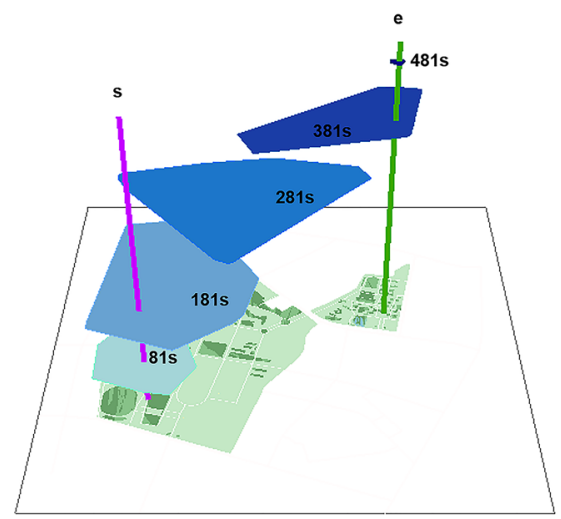

(a)

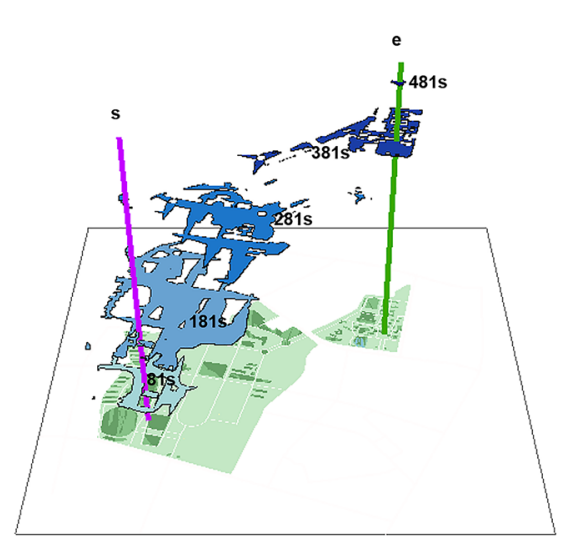

(c)

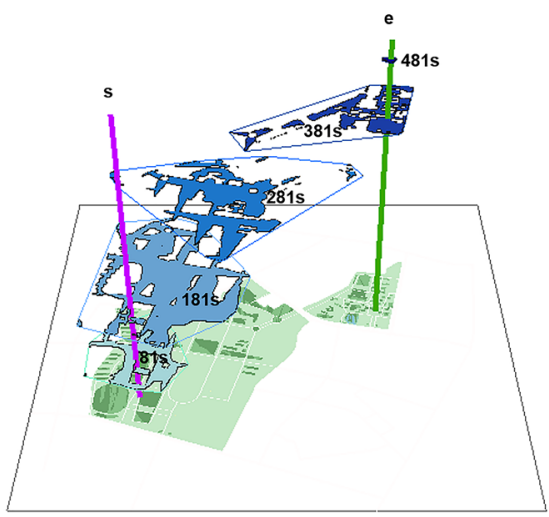

(b)

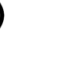


planar disks (Miller and Bridwell 2009) and networked disks (Kuijpers and Othman 2009; Kuijpers et al. 2010; Yin et al. 2020). Such space-time disks reveal, at a glance, the non-uniformity of the time-cost surface, which contrasts sharply with the case of an unconstrained environment, emphasising the impact of accounting for the dual constraints of traffic networks and walking surfaces. This heterogeneity causes the space-time disks to be non-smooth at the boundaries, even appearing hollow inside. Nevertheless, the interior is connected. Finally, the space-time disk, as an outcome of a geographic accessibility analysis of the time-cost surface model, can be used to assess the impact of different spatial and temporal uncertainty factors on accessibility and can be used to analyse the accuracy of the model.

Figure 6 compares two space-time disks corresponding to $81 \mathrm{~s}$ : the blue-filled disk is generated based on a two-layer network, whereas the yellow-filled one is based on a pure raster grid. Figure 6 a shows that the divergence between the blue and yellow disks is manifested in two ways: one is in the blue disk, e.g., Point A is not on the yellow disk, and the other is in the yellow disk, e.g., Point B is not on the blue disk. According to the two-layer network and the shortest path algorithm, $t_{\mathrm{A}}^{-}=78.95 \mathrm{~s}, t_{\mathrm{A}}^{+}=259.18 \mathrm{~s}$, and $t_{\mathrm{B}}^{-}=129.65 \mathrm{~s}, t_{\mathrm{B}}^{+}=180.76 \mathrm{~s}$, and the corresponding least-cost paths between $s$ and $\mathrm{A}$ (or B) and between $\mathrm{A}$ (or $\mathrm{B}$ ) and $e$ are shown in Fig. 6b. As can be seen from this figure, these paths follow the physical road network, except for the walking sections before and after point $\mathrm{B}$ (because there is no road directly connected to point B); consequently, with walking sections of $272 \mathrm{~m}$ in length and highway sections of $4111 \mathrm{~m}$ in length, they can be investigated in the field, which, to a certain extent, verifies the correctness of the reachability of points A (and B) and the validity of the two-layer network model. Again, the STP through point A can be shown in $x-y-t$ space (Fig. 6c); just as the STP through point B in Fig. 6d. Although this comparison is for a single example, the results are representative of the general trends. Overall, as expected, the results of both applications demonstrate that the new technique has the potential to more precisely estimate the space-time accessibility than the pure raster-based field model.

\section{Discussion and conclusions}

The application of layer-based time geography to network- and nonnetworkconstrained travel environments illustrated the technique's potential, both in the context of movement along with a spatial network and across a walking surface. Likewise, the results of its application to urban settings indicate the method is particularly useful for understanding human activities and travel possibilities in space and time. However, beyond this example, this reasoning may apply for many real-world applications, as other network-based traffic (such as rail transit) + walking is also the basic mode of travel. Our approach adds to the earlier work by Miller and Bridwell (2009) who propose a field-based representation implemented as a lattice approximation. Although their approach allows for the dual constraints of the traffic network and walking surface, the physical transportation network cannot be completely represented by the grid model. Our approach preserves the physical transportation network and offers a valuable alternative if 


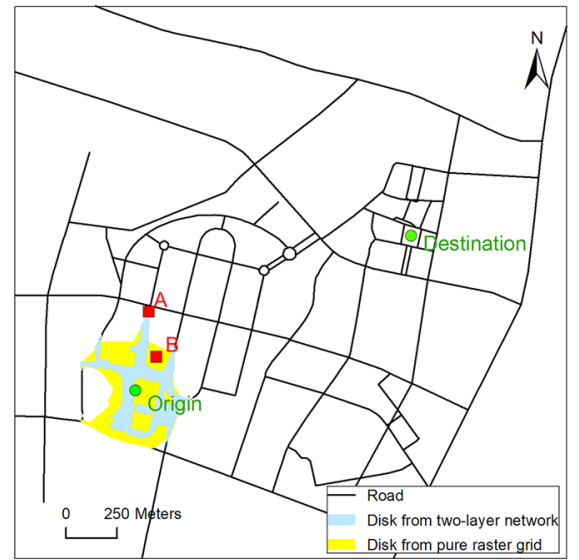

(a)

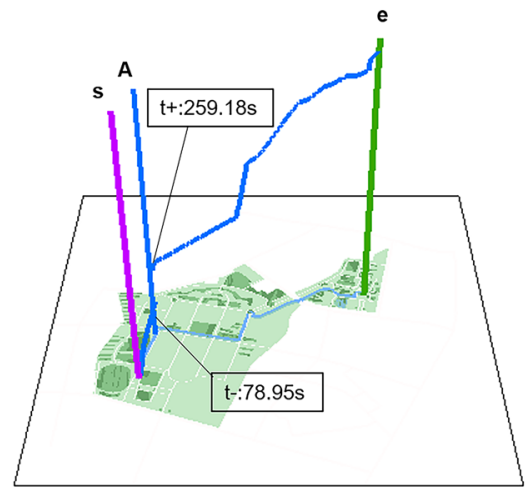

(c)

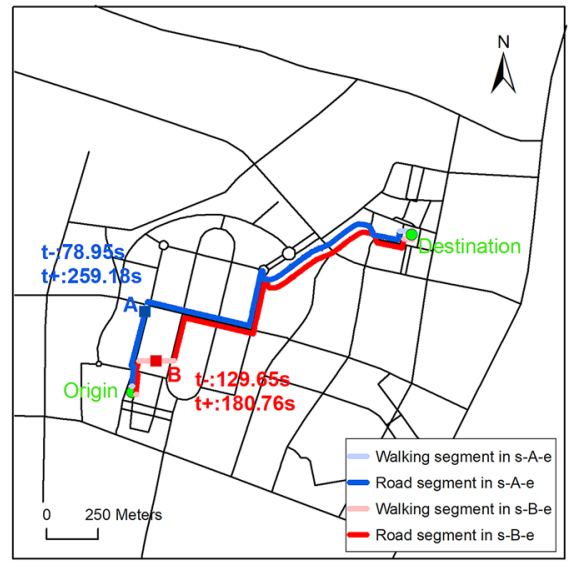

(b)

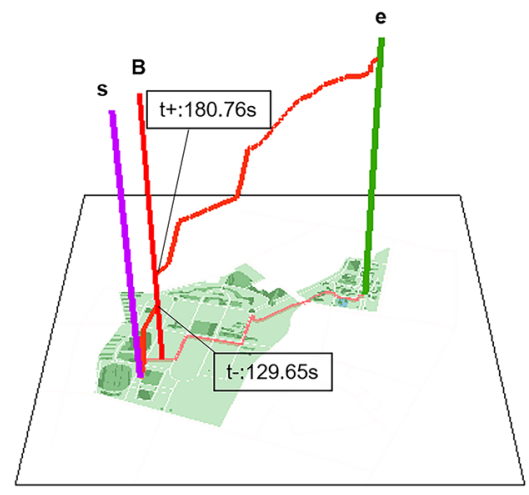

(d)

Fig. 6 Comparison of two space-time disks overlain on the map: a blue and yellow filled disks at $81 \mathrm{~s}$ derived from a two-layer network and pure raster grid, respectively; b two shortest paths passing A and B, respectively; c STP through point A; d STP through point B (color figure online)

the necessary walking surface data are insufficient to build a reliable and fully covering velocity field. These results suggest that layer-based time geography is suitable for modelling the velocity surface of areas involving travel environments.

The two-layer network model, which takes into account area-related traffic modes, can be generalised to the supernetwork model. For example, it can function as a fundamental building block of a supernetwork or as a method to construct supernetworks involving planar areas. Although the representation of the area is not critical when the proportion of the area is very small, it needs to be seriously considered when the proportion is very large. Consequently, the generalisation of the proposed method to an area-related supernetwork can improve the supernetwork model and, consequently, is part of future research. 
Moreover, the proposed model, as an alternative to the raster-based time-geographic field, improves the representation of the time-cost surface by time geography and, thus, can improve the expression of concepts such as space-time paths, prisms, disks, and isochrones (O'Sullivan et al. 2000). These concepts can act as tools for evaluating the service scope of urban facilities, public health, and other characteristics of the urban environment (Fayyaz et al. 2017), as well as constructing Thiessen polygons based on time-cost. To go further, by extension, the chains (or necklaces) of chronologically successive prisms are powerful tools for assessing the consistency of data quality in situations in which the overall quality of individual trajectory data is uncertain (Loraamm et al. 2018), as well as in cases of dynamic interaction (Winter and Yin 2010; Yin et al. 2018). In terms of application, the approach might be useful for studying the transmission of infectious diseases involving space-time accessibility, such as COVID-19. A future study will use this method to construct a prism chain for each COVID-19 patient prior to diagnosis in retrospect the patient's space-time accessibility range and the contacts within this range, providing a theoretical basis for the maximum possible discovery of contacts. These discussions above indicate that the improved model proposed in this article can provide the basis for the construction and application of key concepts of time geography.

Although layer-based time geography outperformed the raster-based variety for the experimental data, there are several considerations that should be noted regarding the technique's performance to guide future research. First, in the two-layer network, the interconnection of the road network and the walking net always occurs at their intersections. For residential streets, these intersections are effective as transfer points, whereas this is not always the case for other roadways (e.g., viaducts, underground tunnels). In addition, whereas the transfer cost of zero performs reasonably well for the time geography that seeks to maximise accessibility, layer-based time geography might be improved using the time expense function of the transfer activity (e.g. Liao 2019). Future work should explore this and other modifications to the transfers.

The second consideration is the non-uniformity of the walking area. In urban space, there are a large number of barriers that can serve as any type of inaccessible area, such as building blocks and water bodies (Delafontaine et al. 2011). Here, the walking area might be cut with buildings or lake boundaries. Therefore, the STP analysis will produce a more meaningful result; once the STP avoids the barriers, areas inside the barriers are not incorporated into the space-time prism. In addition, the walking speed is usually related to the slope of the terrain. Dealing with obstacles and slopes can lead to the following problems: traversable passageways in buildings are ignored and clipping creates isolated areas that should be connected to the surroundings. Consequently, the consideration of indoor passages and avoidance of "islands" may be promising ways to effectively model the walking environment. In addition, the neighbour network and actual trails, which are located inside and outside the walking zone, may not be automatically connected, which reminds us that the construction of the walking net needs to add checks to ensure such connection.

Third, this research assumed that layer-based time geography is most applicable when a planar walking area is so large that it cannot be adequately represented 
using several linear trails. If the actual trails are all along with the road network, a copy of the road network can effectively represent the walking environment or mode. Thus, a recommended approach might include first the application of a supernetwork model based on a copy of the road network to represent the walking mode and then, if the model is too uncertain, the application of layer-based time geography as an alternative.

Fourth, although this article explored the walking zone largely in the context of the raster model, the method may be equally applicable to other logical models as well. Example applications might include the characterisation of the continuous walking zone through network, including triangles and hexagons. Additionally, the technique might be further modified for use with a Voronoi diagram to represent the walking zone as discrete Thiessen polygons. Such a modification might involve determining the set of original discrete points from the nodes of the road network and generating Thiessen polygons as cells and their dual graphs as neighbour networks, Delaunay triangles. In summary, layer-based time geography provides a useful method for generating a velocity surface for network and nonnetwork travel environments; furthermore, the general hierarchical approach can be applied to a variety of other logical models that can replace the raster model.

Finally, parameter selection for layer-based time-geographic implementation exacts a significant impact on results. Specifically, the choice of parameters surrounding the spatial resolution of raster data and the neighbourhood type is highly sensitive in their effect on results and demand in terms of computation. Like for any raster analysis, the calculation of grid cells on the walking area is based on cell centroids (Downs et al. 2014); therefore, results are subject to some spatial generalisation. In mathematics, the discrete raster model tends to a continuous field when the pixel size is small enough, which means that generalisation can be resolved by increasing the lattice density. Yet better precision leads to an increase in data storage and computing demands. Therefore, it is necessary to select a spatial resolution and a neighbourhood type conditioned on the actual situation, such as specific application requirements and computer capabilities. Future research may seek to address a more dynamic or data-driven assignment of parameters for layer-based time-geographic calculations.

Acknowledgements The authors would like to thank the National Key R\&D Program of China (Grant number 2017YFB0503700), (Grant number 2017YFB0503500); the National Science Foundation of China (NSFC) (Grant number 41671381) and (Grant number 41531177) for their support in this research.

Funding This work was supported by the National Key R\&D Program of China (Grant number 2017YFB0503700), (Grant number 2017YFB0503500); the National Science Foundation of China (NSFC) (Grant number 41671381) and (Grant number 41531177).

\section{Compliance with ethical standards}

Conflict of interest The authors declare that they have no conflict of interest. 


\section{References}

Apparicio P, Abdelmajid M, Riva M, Shearmur R (2008) Comparing alternative approaches to measuring the geographical accessibility of urban health services: distance types and aggregationerror issues. Int J Health Geogr 7:7. https://doi.org/10.1186/1476-072X-7-7

Arentze T, Timmermans H (2004) Multistate supernetwork approach to modelling multi-activity, multimodal trip chains. Int J Geogr Inf Sci 18:631-651. https://doi.org/10.1080/136588104100017 01978

Carlier K, Fiorenzo-Catalano S, Lindveld C, Bovy P (2003) A supernetwork approach towards multimodal travel modelling. In: TRB (ed) Proceedings 82nd annual meeting of the Transportation Research Board, Washington DC

Collischonn W, Pilar JV (2000) A direction dependent least-cost-path algorithm for roads and canals. Int J Geograph Inf Sci 14:397-406. https://doi.org/10.1080/13658810050024304

Delafontaine M, Neutens T, Van de Weghe N (2011) Modelling potential movement in constrained travel environments using rough space-time prisms. Int J Geogr Inf Sci 25:1389-1411. https://doi. org/10.1080/13658816.2010.518571

Delamater PL, Messina JP, Shortridge AM, Grady SC (2012) Measuring geographic access to health care: raster and network-based methods. Int J Health Geogr 11:15. https://doi. org/10.1186/1476-072X-11-15

Downs JA, Horner MW (2014) Adaptive-velocity time-geographic density estimation for mapping the potential and probable locations of mobile objects. Environ Plan B Plan Des 41:1006-1021. https:// doi.org/10.1068/b130065p

Downs JA, Lamb D, Hyzer G, Loraamm R, Smith ZJ, O’Neal BM (2014) Quantifying spatio-temporal interactions of animals using probabilistic space-time prisms. Appl Geogr 55:1-8. https://doi. org/10.1016/j.apgeog.2014.08.010

Dykes JA, Mountain DM (2003) Seeking structure in records of spatio-temporal behaviour: visualization issues, efforts and applications. Comput Stat Data Anal 43:581-603. https://doi.org/10.1016/s0167 -9473(02)00294-3

Fayyaz SK, Liu X-YC, Porter RJ (2017) Dynamic transit accessibility and transit gap causality analysis. J Transp Geogr 59:27-39. https://doi.org/10.1016/j.jtrangeo.2017.01.006

Gonçalves AB (2010) An extension of GIS-based least-cost path modelling to the location of wide paths. Int J Geogr Inf Sci 24:983-996. https://doi.org/10.1080/13658810903401016

Goodchild M (1977) An evaluation of lattice solutions to the problem of corridor location. Environ Plan A 9:727-738. https://doi.org/10.1068/a090727

Hägerstraand T (1970) What about people in regional science? Pap Reg Sci 24:7-24. https://doi. org/10.1111/j.1435-5597.1970.tb01464.x

Jordan MI, Jacobs RA (1994) Hierarchical mixtures of experts and the EM algorithm. Neural Comput 6:181-214. https://doi.org/10.1109/ijcnn.1993.716791

Kobayashi T, Miller HJ, Othman W (2011) Analytical methods for error propagation in planar spacetime prisms. J Geogr Syst 13:327-354. https://doi.org/10.1007/s10109-010-0139-z

Kuijpers B, Othman W (2009) Modeling uncertainty of moving objects on road networks via space-time prisms. Int J Geogr Inf Sci 23:1095-1117. https://doi.org/10.1080/13658810802097485

Kuijpers B, Miller HJ, Neutens T, Othman W (2010) Anchor uncertainty and space-time prisms on road networks. Int J Geogr Inf Sci 24:1223-1248. https://doi.org/10.1080/13658810903321339

Kwan M-P, Hong X-D (1998) Network-based constraints-oriented choice set formation using GIS. Geogr Syst 5:139-162

Lee J, Miller HJ (2018) Measuring the impacts of new public transit services on space-time accessibility: an analysis of transit system redesign and new bus rapid transit in Columbus, Ohio, USA. Appl Geogr 93:47-63. https://doi.org/10.1016/j.apgeog.2018.02.012

Leung Y, Zhao Z, Ma J-H (2016) Uncertainty analysis of space-time prisms based on the moment-design method. Int J Geogr Inf Sci 30:1336-1358. https://doi.org/10.1080/13658816.2015.1130830

Liao F-X (2019) Space-time prism bounds of activity programs: a goaldirected search in multi-state supernetworks. Int J Geogr Inf Sci 33:900-921. https://doi.org/10.1080/13658816.2018.1563300

Long J (2016) A field-based time geography for wildlife movement analysis. In: International conference on GIScience short paper Proceedings, vol 1. https://doi.org/10.21433/b3113ht0m7hh

Long JA (2018) Modeling movement probabilities within heterogeneous spatial fields. J Spat Inf Sci 2018:85-116. https://doi.org/10.5311/josis.2018.16.372 
Long JA, Nelson TA (2013) A review of quantitative methods for movement data. Int J Geogr Inf Sci 27:292-318. https://doi.org/10.1080/13658816.2012.682578

Loraamm RW (2019) Incorporating behavior into animal movement modeling: a constrained agentbased model for estimating visit probabilities in space-time prisms. Int J Geogr Inf Sci. https://doi. org/10.1080/13658816.2019.1658875

Loraamm RW, Downs JA, Lamb D (2018) A time-geographic approach to quantifying wildlife-road interactions. Trans GIS 23:70-86. https://doi.org/10.1111/tgis.12497

Miller HJ (1991) Modelling accessibility using space-time prism concepts within geographical information systems. Int J Geogr Inf Syst 5:287-301. https://doi.org/10.1080/02693799108927856

Miller HJ (2005) A measurement theory for time geography. Geogr Anal 37:17-45. https://doi.org/10.11 11/j.1538-4632.2005.00575.x

Miller HJ, Bridwell SA (2009) A field-based theory for time geography. Ann Assoc Am Geogr 99:49-75. https://doi.org/10.1080/00045600802471049

Neutens T, Schwanen T, Witlox F (2011) The prism of everyday life: towards a new research agenda for time geography. Transp Rev 31:25-47. https://doi.org/10.1080/01441647.2010.484153

O'Sullivan D, Morrison A, Shearer J (2000) Using desktop GIS for the investigation of accessibility by public transport: an isochrone approach. Int J Geogr Inf Sci 14:85-104. https://doi. org/10.1080/136588100240976

Sanderson GC (1966) The study of mammal movements: a review. J Wildl Manag. https://doi. org/10.2307/3797914

Shaw S-L, Yu H (2009) A GIS-based time-geographic approach of studying individual activities and interactions in a hybrid physical-virtual space. J Transp Geogr 17:141-149. https://doi. org/10.1016/j.jtrangeo.2008.11.012

Shelat S, Huisman R, van Oort N (2018) Analysing the trip and user characteristics of the combined bicycle and transit mode. Res Transp Econ 69:68-76. https://doi.org/10.1016/j.retrec.2018.07.017

Tobler WR (1993) Non-isotropic geographic modeling. National Center for Geographic Information and Analysis, Santa Barbara

Upchurch C, Kuby M, Zoldak M, Barranda A (2004) Using GIS to generate mutually exclusive service areas linking travel on and off a network. J Transp Geogr 12:23-33. https://doi.org/10.1016/j.jtran geo.2003.10.001

Versichele M, Neutens T, Claeys Bouuaert M, Van de Weghe N (2014) Time-geographic derivation of feasible co-presence opportunities from network-constrained episodic movement data. Trans GIS 18:687-703. https://doi.org/10.1111/tgis.12050

Winter S, Yin Z-C (2010) Directed movements in probabilistic time geography. Int J Geogr Inf Sci 24:1349-1365. https://doi.org/10.1080/13658811003619150

Yin Z-C, Duan Q-Y, Sun H-T, Liu Q-Q, Bao Y, Wang Z-Q (2013) Time geographic network modeling for restraint space of transportation network. In: The fifth international conference on advanced geographic information systems, applications, and services. Nice, France, 24 February 24-1 March 2013, pp 93-98

Yin Z-C, Wu Y, Winter S, Hu L-F, Huang J-J (2018) Random encounters in probabilistic time geography. Int J Geogr Inf Sci 32:1026-1042. https://doi.org/10.1080/13658816.2018.1428748

Yin Z-C, Li S-J, Ying S, Jin Z-H-N, Liu H, Xiao J-Q (2020) Method for calculating the encounter probability in network space. Trans GIS 00:1-21. https://doi.org/10.1111/tgis.12605

Publisher's Note Springer Nature remains neutral with regard to jurisdictional claims in published maps and institutional affiliations. 


\section{Affiliations}

\section{Zhangcai Yin ${ }^{1} \cdot$ Zhanghaonan Jin ${ }^{1}$. Shen Ying ${ }^{2}$ (D) Sanjuan $\mathrm{Li}^{1} \cdot$ Qingquan Liu $^{3}$}

Zhangcai Yin

yinzhangcai@whut.edu.cn

Zhanghaonan Jin

219610@whut.edu.cn

Sanjuan Li

lisanjuan@whut.edu.cn

Qingquan Liu

lqqgis@outlook.com

1 School of Resources and Environmental Engineering, Wuhan University of Technology, Wuhan 430070, China

2 School of Resource and Environmental Sciences, Wuhan University, Wuhan 430061, China

3 China Antarctic Surveying and Mapping Research Center, Wuhan University, Wuhan 430061, China 